\title{
An improved method for the measurement of mechanical properties of bone by nanoindentation
}

\author{
B. Tang ${ }^{1}$, A.H.W. Ngan ${ }^{1 *}$, W. W. Lu $^{2}$. \\ ${ }^{1}$ Department of Mechanical Engineering \\ ${ }^{2}$ Department of Orthopaedics and Traumatology \\ The University of Hong Kong, Pokfulam Road \\ Hong Kong, P.R. China \\ *Correspondence author
}

\begin{abstract}
Nanoindentation is widely used to measure the mechanical properties of bio-tissues. However, viscoelastic effects during the nanoindentation are seldom considered rigorously, although they are in general very significant in bio-tissues. In this study, a recently developed method for correcting the viscoelastic effects during nanoindentation is applied to mice bone samples. This method is found to yield reliable elastic modulus and hardness results from forelimb and femur cortical bone samples of C57 BL/6N and ICR mice. The creep properties of the samples are also characterized by a novel procedure using nanoindentation. The measured mechanical properties correlate well with the calcium content of the bone samples.
\end{abstract}

Keywords: Cortical bone; Nanoindentation; Elastic modulus; Hardness; Viscosity 


\section{Introduction}

In the past, tensile and bending tests have been the most commonly used technique to measure the mechanical properties of hard bio-tissues [1-4]. These techniques can indeed provide useful information on the bulk average mechanical properties of bio-tissues, but local or gradient information due to, for example, the hierarchal structure of the bio-tissues, cannot be obtained. A number of researchers have recently employed nanoindentation to measure the mechanical properties of bio-tissues [5-14]. Nanoindentation is a novel technique originally developed to measure the mechanical properties of micron-scale material volumes such as electronic thin films and small second phase particles. As applied to soft biological tissues, the limitations of this technique have yet to be properly recognized. In particular, nearly all of the nanoindentation studies on bio-tissues reported to-date [5-14] employed the Oliver-Pharr method [15] to obtain elastic modulus and hardness values from the nanoindentation data. The basic assumption involved in this method is that the sample behaves purely elastically during unloading, but biological tissues such as bone are well-known to be viscoelastic in both the macroscopic level [16] as well as the microstructural level [5,9,10,12]. Material viscoelastic effects during unloading are well-known to lead to erroneous results in the estimation of contact stiffness and area using the Oliver-Pharr method [17-24], and in the past, increasing the holding time before unloading and increasing the unloading rate have been suggested as effective procedures to reduce viscoelastic effects during unloading $[12,14,15]$. As applied to very soft materials including most biological tissues, since the severity of the viscoelasticity depends on a complicated convolution of the peak load, the holding duration before unloading and the unloading rate [18], it is seldom known whether a subjective choice of the pre-unloading holding duration and unloading rate can in fact be effective in suppressing viscoelastic effects. To decide on a suitable load scheme that would lead to negligible viscoelastic effects during unloading, one would have to perform a series of trial indentations to attempt to achieve convergence of the calculated modulus and hardness results. These trial indentations have to be performed on locations which are nearby enough to avoid influence from intrinsic material gradients, and yet have to be properly spaced to avoid interference between indentations. Unlike monolithic engineering materials, most biological tissues are highly spatially inhomogeneous with strong gradients and high densities of internal irregularities. The trial-and-error procedure can often be very tedious to perform in practice.

An alternative solution is to allow the viscoelastic effects to occur, but then to 
use a method that has been well established in monolithic engineering materials to correct for the viscoelastic effects. Such a method is now available in the literature $[17,19,21,23]$, and in this study, this method is used to measure the mechanical properties of bone samples from two species of mice, namely, C57 BL/6N and ICR. Rather than attempting to understand the difference in the bone properties in these two types of mice, this study focuses more on illustrating the applicability and importance of the viscoelasticity correction procedure in measuring soft bio-tissues. In the following, a brief review of the nanoindentation methodology is first given, followed by experimental procedures and results in later sections.

\section{Brief Review of Nanoindentation Methodology}

In depth-sensing indentation, displacement and force data are collected continuously during the tip-sample contact period, which are then analyzed to obtain mechanical properties such as the elastic modulus and hardness of the sample. The most standard analysis method is due to Oliver and Pharr [15] and is based on Sneddon's solution for the problem of elastic contact between a conical tip and a flat surface [25]. In this method, the first step is to fit the load-displacement curve during the unloading period by a power-law equation to find the elastic contact stiffness at the onset of unload, defined as $S=d P / d h$, where $P$ is load and $h$ is indenter displacement. The contact depth $h_{c}$ at the onset of unload is then calculated as

$$
h_{c}=h_{m}-\varepsilon \frac{P_{m}}{S},
$$

where $h_{m}$ and $P_{m}$ are the tip displacement and load at the onset of unload respectively, and $\varepsilon$ is a constant, which for a Beckovich tip is 0.75 . The tip-sample contact area $A_{\mathrm{c}}$ can then be calculated from the contact depth by the tip's calibrated area function $A_{c}=f\left(h_{c}\right)$. After $A_{c}$ is known, the reduced modulus $E_{r}$ can be calculated from

$$
E_{r}=\frac{\sqrt{\pi}}{2} \frac{S}{\sqrt{A_{c}}} .
$$

The Young's modulus of the sample $E$ is given by

$$
\frac{1}{E_{r}}=\frac{\left(1-v^{2}\right)}{E}+\frac{\left(1-v_{i}^{2}\right)}{E_{i}},
$$


where $E_{i}$ is Young's modulus of the tip, $v$ and $v_{i}$ are the Poisson ratio of the sample and tip respectively. The contact hardness, defined as the mean pressure that the material will support under load, is given by

$$
H=\frac{P_{m}}{A_{c}}
$$

The Oliver-Pharr method is based on the assumption that the tip-sample contact is purely elastic during unloading. On the other hand, bone is well-known to be viscoelastic $[5,9,10,12,14]$, and for viscoelastic solids, the Oliver-Pharr method is known to be inappropriate $[18,19,21,24]$. Ngan and co-workers [17,19,21,23] have shown that the viscoelastic effects during nanoindentation can be corrected by a rather simple method. By assuming a general power-law viscoelastic model of the Maxwell type, it was shown that the real elastic contact stiffness $S_{\mathrm{e}}$ is related to the apparent unloading stiffness $S=d P / d h$ by

$$
\frac{1}{S_{e}}=\left(\frac{1}{S}-\frac{\dot{h}_{h}}{\dot{P}_{u}}\right) \frac{1}{\left(1-\dot{P}_{h} / \dot{P}_{u}\right)},
$$

where $\dot{P}_{h}$ and $\dot{h}_{h}$ are respectively the loading rate and displacement rate just before the unloading, and $\dot{P}_{u}$ is the unloading rate just after the step change [21,23]. Substituting $S_{e}$ for $S$ in eqn. (1) and (2) can effectively remove viscoelastic effects on the calculated reduced modulus and tip-sample contact depth and hence can improve the accuracy of the measured elastic modulus and hardness. In eqn. (5), $\dot{P}_{h}$ is close to zero if the nanoindentation test is conducted with a feedback loop to control the load at the preset value during the holding period at maximum load. In the absence of such a feedback control, the actual load applied on the sample may drop significantly, as a result of the increasing spring forces holding the indenter as the sample creeps under the peak load [21-23,25]. In this case, viscoelasticity effects can still be corrected if the actual recorded value of $\dot{P}_{h}$ is used in eqn. (5).

In addition to elastic modulus and nanohardness, the creep component of the deformation can also be measured by the nanoindentation technique. In our recent work in 2004 [23], an improved method to measured the viscosity of materials using 
nanoindentation was proposed. It was found that the viscosity $\eta$ of materials can be measured by

$$
\eta=\frac{E_{r} P_{h}}{4\left(S_{e} \dot{h}_{h}-\dot{P}_{h}\right)} .
$$

Here $\dot{P}_{h}, P_{h}$ and $\dot{h}_{h}$ are respectively the load rate, load and the tip displacement rate at the end of the load hold. $E_{r}$ is the reduced modulus, and $S_{\mathrm{e}}$ is the real elastic stiffness calculated from eqn. (5).

\section{Experimental Details}

\subsection{Sample Preparation}

To investigate the applicability of the viscoelasticity correction method to bio-tissues, bone samples were collected from two $\mathrm{C} 57 \mathrm{BL} / 6 \mathrm{~N}$ mice and an ICR mouse. Four forelimb bone samples were collected from one C57 mouse, and are labeled as FC-1 to FC-4. After removing from the mouse by scalpel, these 4 forelimb bone samples were mounted in a single cylindrical brass block by unsaturated polyester resin. The reason for mounting the four samples into a common brass block is to enable the samples to be treated together subsequently, so that they have as similar conditions as is possible. Brass was chosen to be the material for the block because of its high thermal conductivity, so that the block can quickly dissipate the heat generated during the polymerization reaction of the mounting resin to minimize the effect of temperature changes on the samples. To estimate the temperature change during the polymerization reaction of the mounting resin, a control experiment was conducted in which a small alcohol thermometer of a similar size to the bone samples was embedded inside an identical hole in a similar brass block by the same polyester resin. The thermometer recorded a temperature change of only about 1 to $2{ }^{\circ} \mathrm{C}$ during the whole polymerization reaction of the resin. The temperature change during the mounting of the bone samples was therefore very minimal. The brass block together with the forelimb samples were mechanically polished using 400 and 800-grit silicon carbide paper, followed by lapping with $6 \mu \mathrm{m}$ and $1 \mu \mathrm{m}$ diamond slurry, and finally finished with $0.3 \mu \mathrm{m}$ alumina solution to produce suitable surface finish for 
nanoindentation tests. The polished samples were ultrasonically cleaned to remove the alumina powder. In this study, all the specimens were tested in dry condition, as was the case in a few previous studies [5,6,7,12]. However, as reported by Rho and Pharr [12], the degree of the wetness on a sample is a varying factor for the mechanical properties measured by nanoindentation. To obtain a uniform condition, the cleaned sample was dried at $24{ }^{\circ} \mathrm{C}$ in air at a relative humidity of $65 \%$ for about $48 \mathrm{~h}$ before nanoindentation tests. In the dried condition, the water content of the bone samples would not match their in vivo conditions, but this is not an important concern here since the aim of the present work is only to illustrate the applicability of the viscoelasticity correction procedure on prototypic bio-tissues.

Two femur bone samples from another C57 mouse and another two from an ICR mouse were also used in the present experiment and are labeled as ICR-1, ICR-2, C57-1, C57-2 hereafter. These four femur samples were carefully removed from the soft tissue by scalpel, and were then cleaned thoroughly in distilled water. Finally, the cleaned bones were embedded into a single cylindrical brass block and were treated using a similar procedure described above for the forelimb samples.

\subsection{Nanoindentation}

The nanoindenter used is a nanohardness tester supplied by CSM Instruments SA in Switzerland. The indenter tip used was a diamond Berkovich tip with Young's modulus $E_{i}$ of $1140 \mathrm{GPa}$ and Poisson ratio $v_{i}$ of 0.07 . In calculating the modulus values from the nanoindentation data, the Poisson ratio for mouse bone is assumed to be 0.3. To systematically compare the results from different locations of the same sample or different samples, there was therefore a need to standardize the peak load. Fig. 1 shows an independent experiment with a step-increasing loading history on the ICR-2 femur sample. It was found that when the peak load increased from $25 \mathrm{mN}$ to $300 \mathrm{mN}$, even after viscoelastic correction, the measured Young's modulus decreased from about 17.7 GPa to 6.8 GPa. Such an indentation size effect is believed to be due to the hierarchal bone structure, i.e. the tip under different loads probes different levels in the hierarchal structure. Hence, in this study, all the tests were conducted at the same peak load of $10 \mathrm{mN}$. On the transverse section of each forelimb cortical bone samples FC-1 to FC-4, nanoindentation tests were performed on 4 randomly selected positions. A multiple-cycle loading schedule was used in the nanoindentation of the forelimb samples. This consisted of 7 loading cycles, and in each loading cycle, the load was first ramped up at a certain rate to reach the peak value of $10 \mathrm{mN}$, followed 
by holding at the peak load for a certain holding time, and then by unloading to 0.1 $\mathrm{mN}$ (or to zero load in the last cycle) with different unloading rates. In each cycle, the peak load, the loading rate and the holding time were $10 \mathrm{mN}, 20 \mathrm{mN} / \mathrm{min}$ and $30 \mathrm{~s}$ respectively, and the unloading rates used in the seven cycles were respectively 1 $\mathrm{mN} / \mathrm{min}, 0.5 \mathrm{mN} / \mathrm{min}, 2 \mathrm{mN} / \mathrm{min}, 5 \mathrm{mN} / \mathrm{min}, 10 \mathrm{mN} / \mathrm{min}, 20 \mathrm{mN} / \mathrm{min}$ and $40 \mathrm{mN} / \mathrm{min}$. In all the nanoindentation tests performed on FC-1 to FC-4 sample, the indent depth recorded at the onset of unloading in each cycle ranged from about $750 \mathrm{~nm}$ to 1250 $\mathrm{nm}$. These depth values indicate that all the tests were performed on the lamellar level of the bone, and so the hierarchal bone structure should not influence significantly the measured mechanical properties.

On the femur bone samples, a simpler loading scheme was used which consisted of a loading stage at a constant rate of $20 \mathrm{mN} / \mathrm{min}$ to a peak load of $10 \mathrm{mN}$, a holding period of 50s, and then unloading. The unloading rates for all the tests were 20 $\mathrm{mN} / \mathrm{min}$ except in 2 tests performed on ICR-1 and C57-1 in which the unloading rate was deliberately set to a much lower value of $1 \mathrm{mN} / \mathrm{min}$ to investigate the influence of viscoelastic effects during the measurement. A low-load hold at $0.1 \mathrm{mN}$ for $60 \mathrm{~s}$ was performed on all the tests before final unloading to determine the thermal drift rate. On each femur sample, 4 areas were selected on the transverse section, and on each selected area, 8 tests were done on the cortical bone. Fig. 2 shows an overview of these four polished femur samples and the locations of the selected test areas. All the tests were performed within $24 \mathrm{~h}$ to minimize the influence that might be caused by environmental changes.

\subsection{SEM Analysis}

The femur samples after the nanoindentation tests were analyzed for compositions using EDAX in a Cambridge S360 scanning electron microscope (SEM). EDAX was done on the untreated bone samples, and after this, the femur samples were sputter-coated with a layer of Au-Pt alloy of about $10 \mathrm{~nm}$ thick for SEM imaging. Fig. 3 shows an SEM image taken from the ICR-1 femur bone sample. In this image, cracks are seen, and because they have rather random orientation and length, they are unlikely to be generated during the indentation process, since indentation fracture would exhibit specific crack geometries related to the indent [27]. These cracks are likely to be caused by the mechanical polishing procedure prior to nanoindentation test. To exclude the possibility of the existing cracks influencing the accuracy of the property measurements, each indent was imaged under the SEM and those indents 
made on a crack or at a distance shorter that twice the indent size from a crack were excluded from the analysis. For instance, the data from the indent 4, 5, 7 in fig. 3 were not included in the analysis.

\section{Results}

After the nanoindentation tests, the collected data were analyzed by the viscoelasticity correction method involving eqn. (5). Fig. 4 shows the calculated elastic modulus for each unloading cycle at different positions of the forelimb samples. It can be seen that the elastic moduli calculated from the different unloading cycles at the same specimen position are rather constant after viscoelasticity correction.

Fig. 5 shows the hardness values of the forelimb samples. It can be seen that the hardness at each position of the forelimb samples after viscoelasticity correction tends to decrease in later load cycles. Since all the cycles had the same peak load of $10 \mathrm{mN}$, the decreasing hardness is simply due to the continuous increase in the tip-sample contact area as the sample crept under load.

The mechanical properties of the ICR and C57 mice femur samples were further investigated. Table 1 presents the elastic modulus calculated from 38 valid nanoindentation tests. The nanoindentation test data obtained after viscoelasticity correction indicate that the ICR femur cortical bone sample has a higher elastic modulus than the C57 femur cortical bone sample.

In a constantly creeping sample, the hardness in general will change with the loading history, and so in the present study, the same loading history prior to unloading was used in all the tests. The hardness results presented in Table 1 indicate that the ICR femur cortical bone has higher hardness than the C57 femur cortical. Table 1 also shows the hardness data obtained by measuring the residual indent areas from the SEM images of the indents. It was found that the hardness values of the ICR femur samples calculated this way are also higher than those of the C57 femur samples. It should be noted that the hardness values calculated by the Oliver-Pharr scheme after viscoelasticity correction are supposed to be the values of load divided by the projected tip-sample contact area under the application of the load, but the hardness values obtained by direct SEM imaging of the indents are values of the load divided by the residual area of the indents after load removal. The elastic recovery of the indent upon load removal is believed to be responsible for the significantly higher 
values of the hardness obtained by SEM imaging as compared to those calculated from the Oliver-Pharr scheme with viscoelasticity correction.

Fig. 6 shows the viscosity measured from the femur samples using eqn. (6). It was found that the ICR femur bone has larger viscosity than the C57 femur bone. In other words, apart from being stiffer and stronger as shown table 2 respectively, the ICR femur bone also creeps more slowly than the C57 femur bone. The EDAX results of each femur sample are shown in Table 2. It was found that the ratio of $\mathrm{Ca}: \mathrm{P}$ is about 1.9 in the ICR femur, while that in the C57 femur is only about 1.1.

\section{Discussion}

The current work demonstrates that it is possible to use an extended method based on the simple Oliver-Pharr analysis to measure the mechanical properties of bone samples which are highly viscoelastic. This procedure is a simple alternative to the more established method of measuring the complex modulus by force modulation and noting the frequency response of the displacement in nanoindentation [28,29]. While it is well known that the complex modulus of a viscoelastic material is a strong function of the test frequency, the present approach is able to yield estimates of elastic modulus and viscosity in a quasi-static load scheme so that frequency would not be an intervening factor.

The results in Table 1 show that the ICR bone sample used in this work has higher Young's modulus and hardness than the C57 sample used. Fig. 6 also indicates that the viscosity of the ICR bone sample is higher than that of the C57 sample. The EDAX results shown in Table 2 indicate that the ICR sample contained higher calcium content than the C57 sample. The EDAX results in Table 2 in fact correspond well to the mechanical results in Table 1 and fig. 6, because in general, bone properties will improve if the calcium content is higher. While it is evident that the two bone samples studied in this work had significantly different mechanical properties and calcium contents, it is important to note that the present results should not be treated as indicative of the generic difference between the two mouse species of $\mathrm{C} 57 \mathrm{BL} / 6 \mathrm{~N}$ and ICR. The reason is that the gender and age of the mice samples used in the present study, unfortunately, were not tracked. To allow for a systematic comparison, a full range of samples with different gender and age should be compared but this is outside the immediate scope of the present investigation, which is simply to illustrate the applicability and importance of the viscoelasticity correction method in nanoindentation measurements of hard bio-tissues. 


\section{Conclusions}

A novel method to correct for the viscoelastic effects during nanoindentation tests on bio-tissue was applied to measure the elastic modulus and hardness of cortical bone samples taken from an ICR mouse and two C57 mice. The viscosity of the mouse bone samples was also measured using a novel procedure in nanoindentation. It was found that the ICR femur sample had higher elastic modulus, hardness and viscosity than the C57 sample. Chemical measurement also shows that the ICR femur sample contained higher calcium content than the C57 femur sample. The results show that viscoelastic correction is necessary to achieve accurate measurement using nanoindentation, especially if the unloading rate is slow.

\section{Acknowledgments}

The work described in this paper was supported by a grant from the Research Grants Council of the Hong Kong Special Administration Region, P. R. China (Project no. HKU7194/04E). We also thank Dr. K.M.C. Cheung for helpful discussions.

\section{References}

[1] C. Milgrom, A. FInESTONE, A. HAMEL, V. MANDES, D. BURR and N. SHARKEY, J. Biomech. 37 (2004) 947.

[2] E. R. C. DRAPER and A. E. GOODSHIP, J. Biomech. 36 (2003) 1497.

[3] C. M. FLAHIFF, D. A. NARMONEVA, J. L. HUEBNER, V. B. KRAUS, F. GUILAK and L. A. SETTON, J. Biomech. 35 (2002) 1285.

[4] D. VASHISHTH, G. J. GIBSON, J. I. KHOURY, M. B. SCHAFFLER, J. KIMURA and D. P. FYHRIE, Bone 28 (2001) 195.

[5] X. M. WANG, F. Z. CUI, J. GE, Y. ZHANG and C. MA, Biomaterials 23 (2002) 4557.

[6] S. HENGSBERGER, A. KULIK and P. H. ZYSSET, Bone 30 (2002) 178.

[7] J. Y. RHO, T. Y. TSUI and G. M. PHARR, Biomaterials 18 (1997) 1325.

[8] C. H. TURNER, J. Y. RHO, Y. TAKANO, T. Y. TSUI and G. M. PHARR, J. Biomech. 32 (1999) 437.

[9] J. Y. RHO, P. ZIOUPOS, J. D. CURREY and G. M. PHARR, J. Biomech. 35 
(2002) 189.

[10] C. E. HOFFLER, K. E. MOORE, K. KOZLOFF, P. K. ZYSSET, M. B. BROWN and S. A. GOLDSTEIN, Bone 26 (2000) 603-609.

[11] Y. ZHANG, F. Z. CUI, X. M. WANG, Q. L. FENG and X. D. ZHU, Bone 30 (2002) 541.

[12] J. Y. RHO and G. M. PHARR, J. Mater. Sci. Mater. Med. 10 (1999) 485.

[13] P. K. ZYSSET, X. E. GUO, C. E. HOFFLER, K. E. MOORE and S. A. GOLDSTEIN, J. Biomech. 32 (1999) 1005.

[14] A. J. BUSYBY, V. L. FERGUSON and A. BOYDE, J. Mat. Res. 19 (2004) 249.

[15] W. C. OLIVER and G. M. PHARR, J. Mat. Res. 7 (1992) 1564.

[16] R. LAKES and S. SAHA, J. Biomech. Eng. 102 (1980) 178.

[17] G. FENG and A. H. W. NGAN, J. Mat. Res. 17 (2002) 660.

[18] A. H. W. NGAN and B. TANG, J. Mat. Res. 17 (2002) 2604.

[19] B. TANG and A. H. W. NGAN, J. Mat. Res. 18 (2003) 1141.

[20] T. CHUDUBA and F. RICHTER, Sur. Coat. Tech. 148 (2001) 191.

[21] A. H. W. NGAN, H. T. WANG, B. TANG and K. Y. SZE, Int. J. Solids Struct. 42 (2005) 1831.

[22] G. FENG and A. H. W. NGAN, Scripta Mater. 45 (2001) 971.

[23] B. TANG and A. H. W. NGAN, Soft Mat. 2 (2004) 125.

[24] Y. T. CHENG, W. Y. NI and C. M. CHENG, J. Mat. Res. 20 (2005) 3061.

[25] B. TANG and A. H. W. NGAN, Soft Mat. 2 (2004) 183

[26] I. SNEDDON, Int. J. Eng. Sci. 3 (1965) 47.

[27] B. LAWN, in "Fracture of brittle solids" (Cambridge University, Cambridge, UK, 1993) p. 249.

[28] J. L. LOUBET, W. C. OLIVER and B. N. LUCAS, J. Mater. Res. 15 (2000) 1195.

[29] A. CHAKRAVARTULA and K. KOMVOPOULOS, Appl. Phys. Lett. 88 (2006) 131901. 
Table 1. Mechanical properties of ICR and C57 femur cortical bone. "n” is the sample size.

\begin{tabular}{|c|c|c|c|c|}
\hline & \multicolumn{2}{|c|}{ ICR cortical bone } & \multicolumn{2}{c|}{ C57 cortical bone } \\
\hline Unloading rate & $20 \mathrm{mN} / \mathrm{min}(\mathrm{n}=19)$ & $1 \mathrm{mN} / \mathrm{min}(\mathrm{n}=1)$ & $20 \mathrm{mN} / \mathrm{min}(\mathrm{n}=17)$ & $1 \mathrm{mN} / \mathrm{min}(\mathrm{n}=1)$ \\
\hline $\mathrm{E}_{\mathrm{VC}}(\mathrm{GPa})$ & $22.09 \pm 0.71$ & 24.76 & $14.22 \pm 2.61$ & 14.11 \\
\hline $\mathrm{H}_{\mathrm{VC}}(\mathrm{MPa})$ & $872.11 \pm 60.10$ & 1081.54 & $592.13 \pm 172.11$ & 499.78 \\
\hline $\mathrm{H}_{\mathrm{SEM}}(\mathrm{MPa})$ & \multicolumn{2}{|c|}{$1518.4 \pm 415.68(\mathrm{n}=20)$} & \multicolumn{2}{|c|}{$785.08 \pm 134.68(\mathrm{n}=18)$} \\
\hline
\end{tabular}

Key: $\mathrm{E}_{\mathrm{VC}}=$ elastic modulus measured by viscoelasticity correction method. $\mathrm{H}_{\mathrm{VC}}=$ hardness measured by viscoelasticity correction method.

$\mathrm{H}_{\mathrm{SEM}}=$ hardness measured by direct SEM imaging of the residual indents.

Table 2. Ca and P contents of femur samples determined by EDAX.

\begin{tabular}{|r|l|l|l|}
\hline Sample & Element & Element \% & Ca: P \\
\hline \multirow{2}{*}{ ICR 1 } & $\mathrm{Ca}$ & 66.0 & \multirow{2}{*}{1.94} \\
\cline { 2 - 3 } & $\mathrm{P}$ & 34.0 & \\
\hline \multirow{2}{*}{ ICR 2 } & $\mathrm{Ca}$ & 66.2 & \multirow{2}{*}{1.96} \\
\cline { 2 - 3 } & $\mathrm{P}$ & 33.8 & \\
\hline \multirow{2}{*}{ C57 1 } & $\mathrm{Ca}$ & 52.4 & \multirow{2}{*}{1.10} \\
\cline { 2 - 3 } & $\mathrm{P}$ & 47.6 & \multirow{2}{*}{1.14} \\
\hline \multirow{2}{*}{ C57 2 } & $\mathrm{Ca}$ & 53.1 & \\
\cline { 2 - 3 } & $\mathrm{P}$ & 46.8 & \\
\hline
\end{tabular}




\section{Figure Captions}

Figure 1. The calculated elastic moduli on the same position of the ICR-2 femur sample at increasing indent depths.

Figure 2. Optical microscopy images of the four femur samples. The square areas are areas selected for nanoindentation.

Figure 3. SEM image of one region of the transverse section of the ICR-1 femur sample.

Figure 4. The elastic modulus of forelimb samples measured after viscoelasticity correction. In each forelimb specimen, indentations were performed at 4 different positions (labeled as 1 to 4 ) using a multi-cycle load schedule described in the text.

Figure 5. The hardness of forelimb samples measured after viscoelasticity correction.

Figure 6. Viscosity measured from the femur samples. The mean viscosity values for the ICR and C57 bone are $2.12 \times 10^{12} \pm 3.18 \times 10^{11} \mathrm{~Pa}$-s and $1.16 \times 10^{12}$ $\pm 5.5 \times 10^{11} \mathrm{~Pa}$-s respectively. 


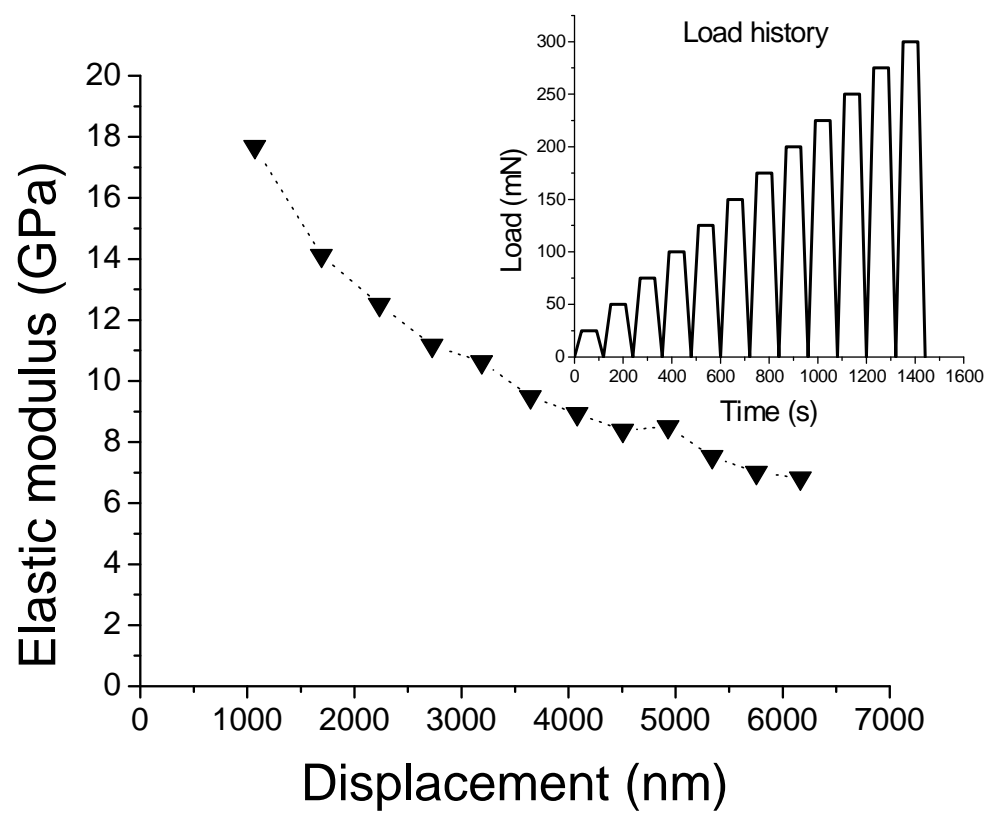

Figure 1 


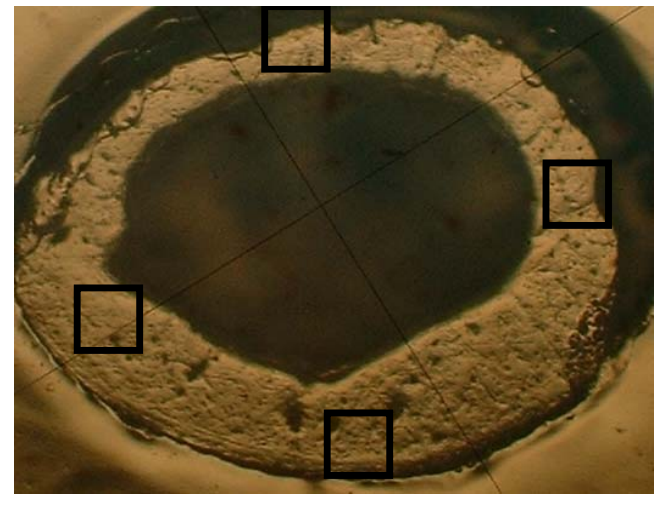

ICR-1

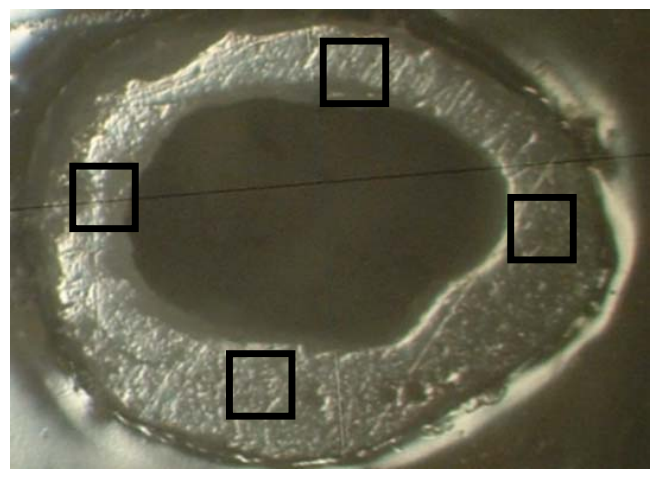

ICR-2

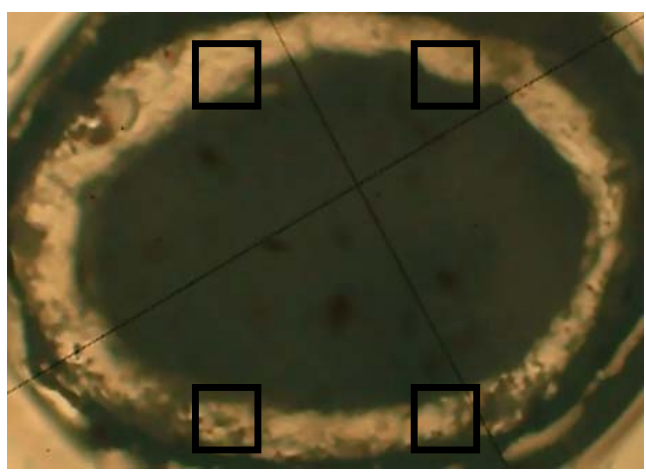

C57-1

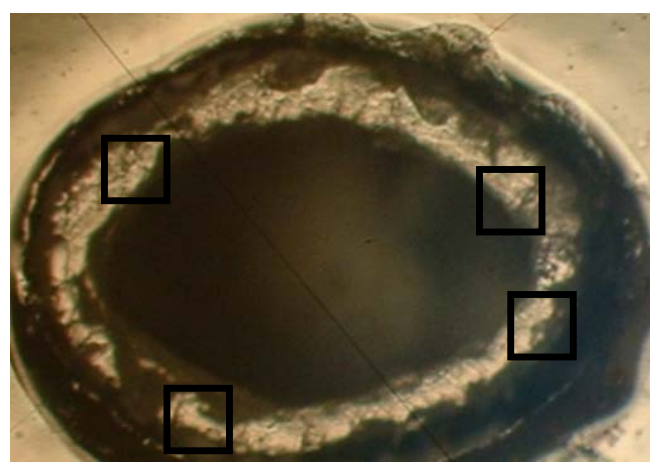

C57-2

Figure 2 


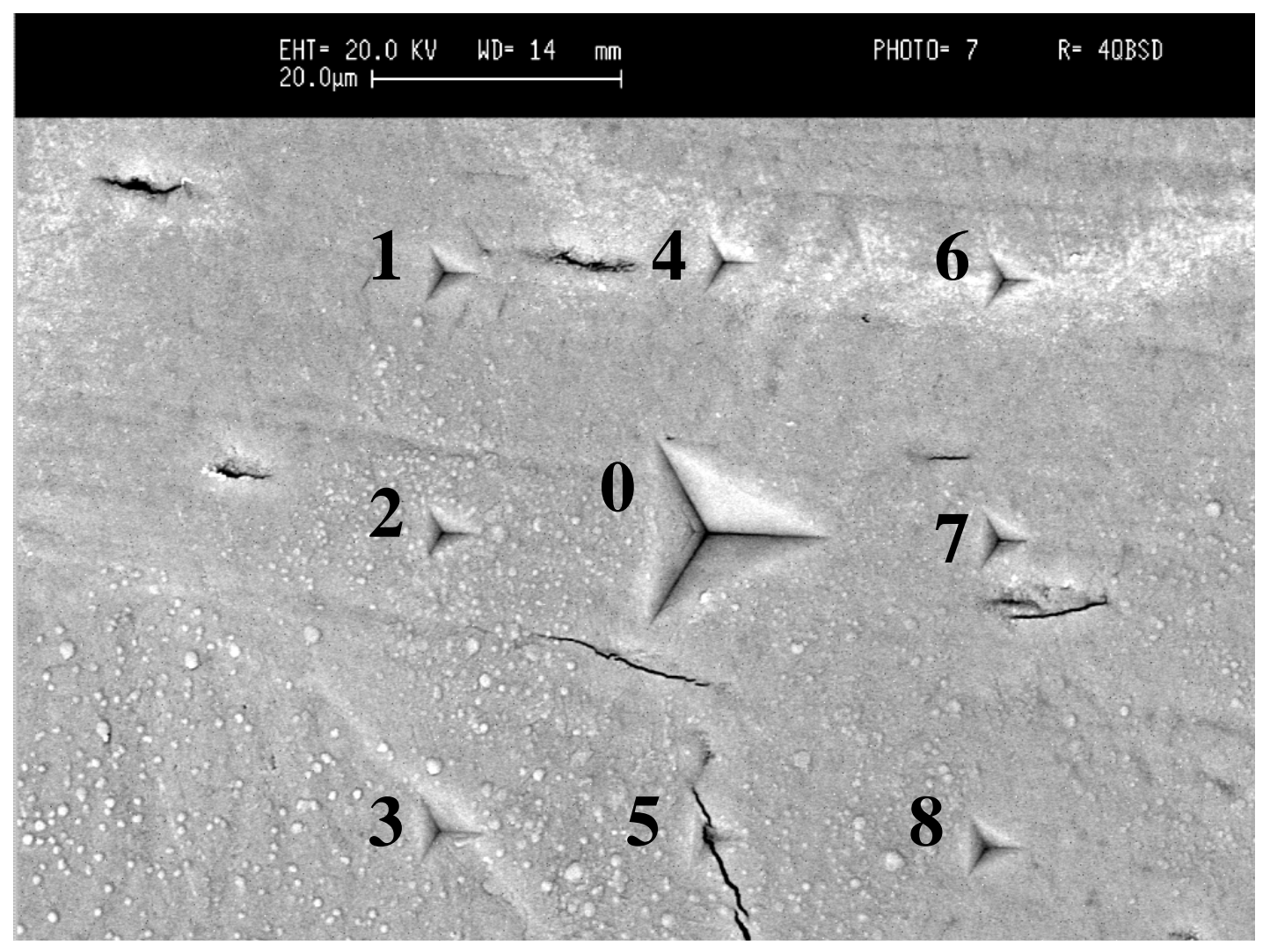

Figure 3 


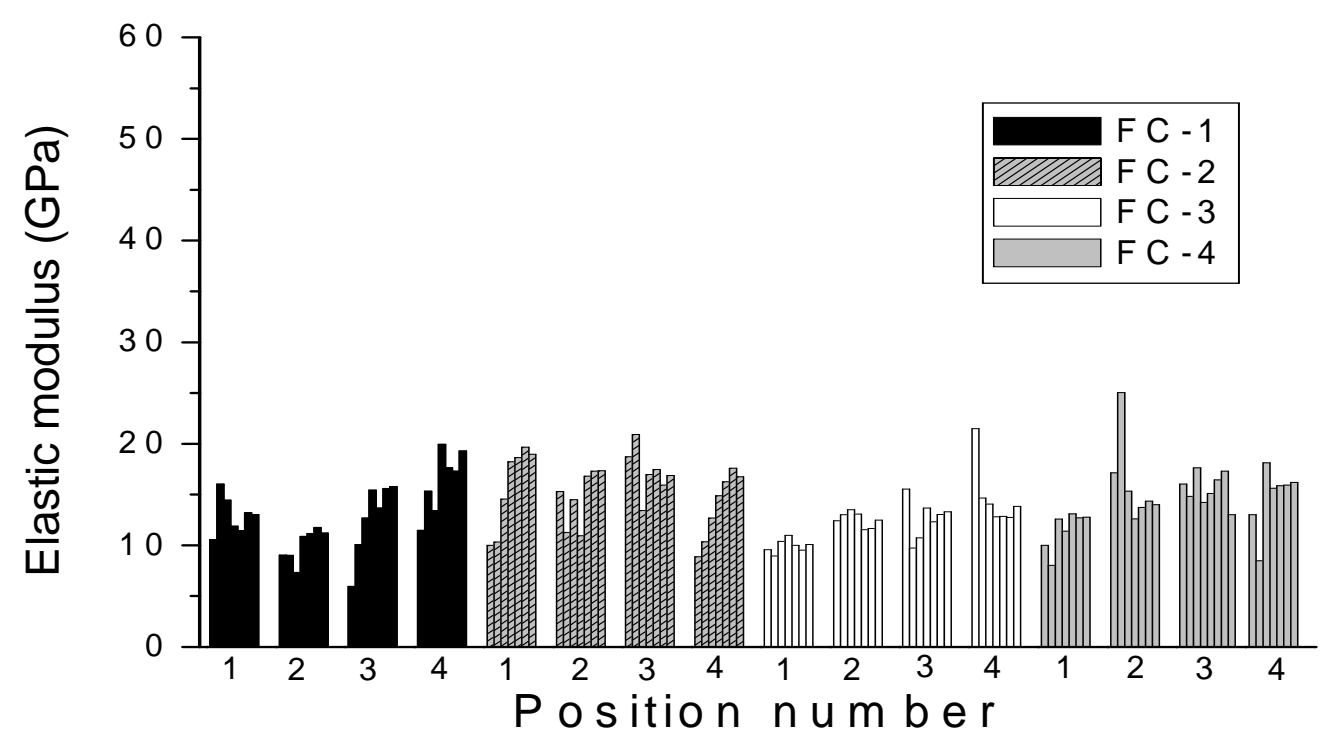

Figure 4

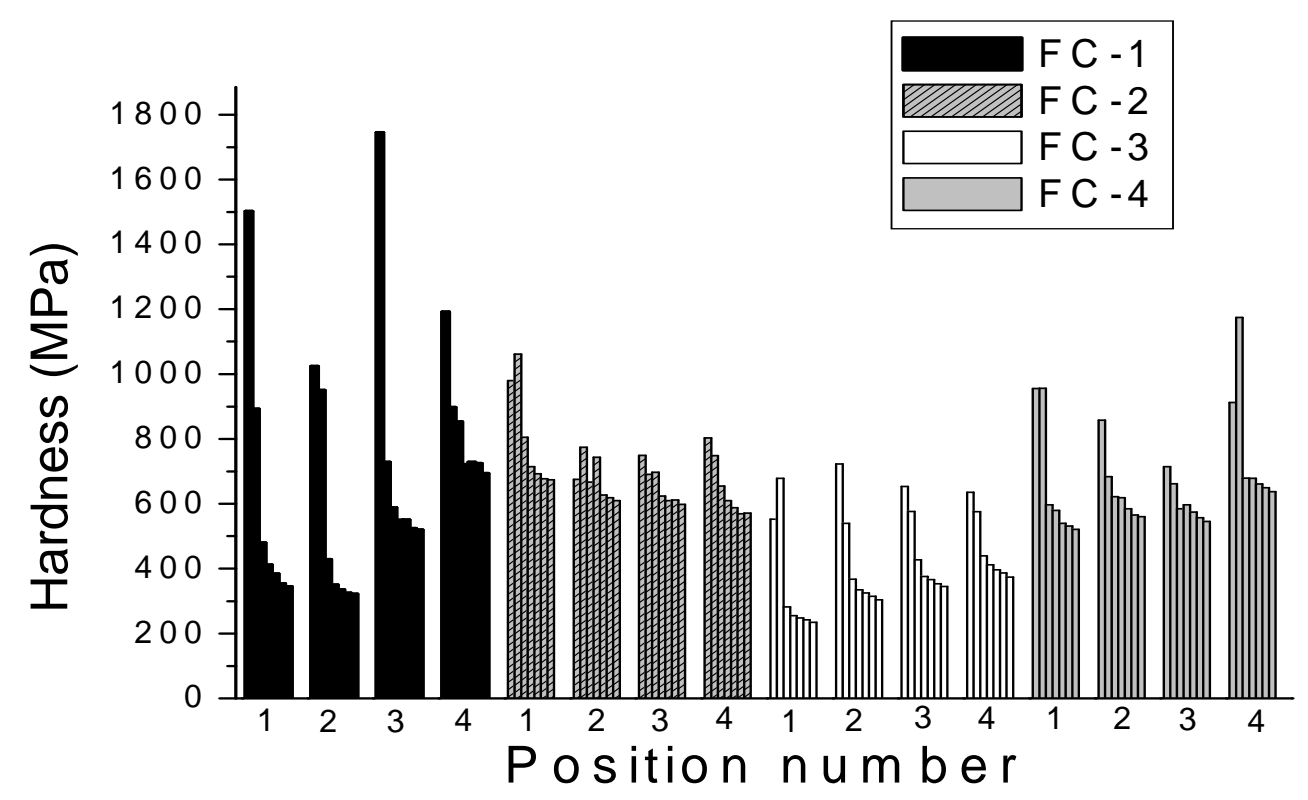

Figure 5 


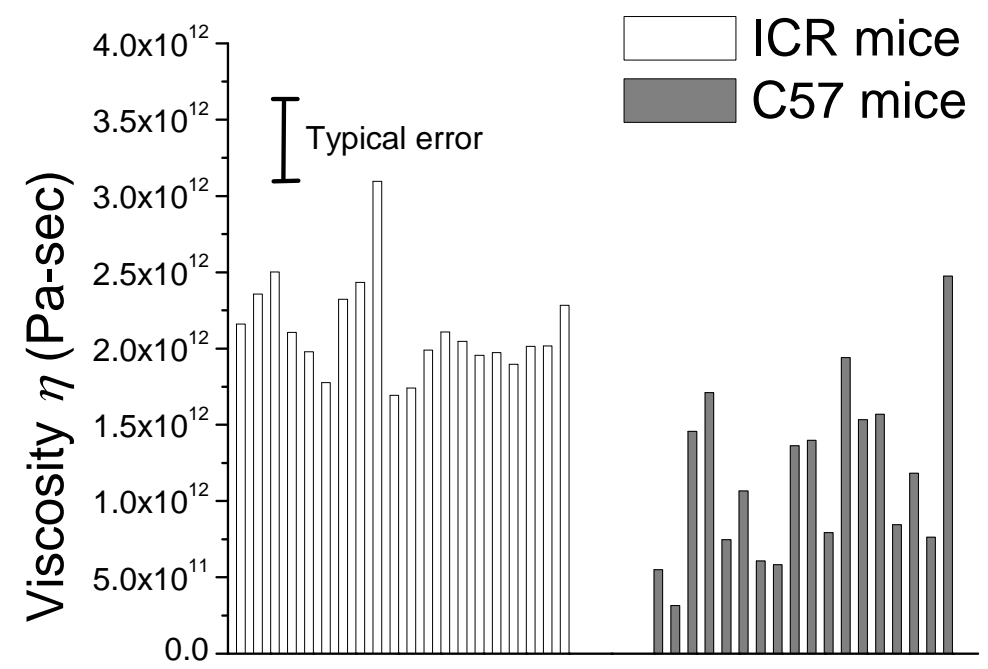

Figure 6 